\title{
Marginality, dividends, and the value in games with externalities
}

Frank Huettner, ESMT Berlin

André Casajus, HHL Leipzig Graduate School of Management 


\title{
Marginality, dividends, and the value in games with externalities ${ }^{\text {th }}$
}

\author{
Frank Huettner ${ }^{\mathrm{a}, *}$, André Casajus ${ }^{\mathrm{b}, \mathrm{c}}$ \\ ${ }^{a}$ ESMT European School of Management and Technology, Schlossplatz 1, 10178 Berlin, Germany \\ ${ }^{b}$ HHL Leipzig Graduate School of Management, Jahnallee 59, 04109 Leipzig, Germany \\ ${ }^{c}$ Dr. Hops Craft Beer Bar, Eichendorffstr. 7, 04277 Leipzig, Germany
}

\begin{abstract}
In the absence of externalities, marginality is equivalent to an independence property that rests on Harsanyi's dividends. These dividends identify the surplus inherent to each coalition. Independence states that a player's payoff stays the same if only dividends of coalitions to which this player does not belong to change. We introduce notions of marginality and independence for games with externalities. We measure a player's contribution in an embedded coalition by the change in the worth of this coalition that results when the player is removed from the game. We provide a characterization result using efficiency, anonymity, and marginality or independence, which generalizes Young's characterization of the Shapley value. An application of our result yields a new characterization of the solution put forth by Macho-Stadler et al. (J Econ Theor, 135, 2007, 339-356) without linearity, as well as for almost all generalizations put forth in the literature. The introduced method also allows us to investigate egalitarian solutions and to reveal how accounting for externalities may result in a deviation from the Shapley value. This is exemplified with a new solution that is designed in a way to not reward external effects, while at the same time it cannot be assumed that any partition is the default partition.
\end{abstract}

Keywords: Shapley value, potential, restriction operator, partition function form game, externalities

2010 MSC: 91A12, JEL: C71, D60

\section{Introduction}

A large part of the literature on cooperative game theory circles around the notion of marginality and its relation to the Shapley value. Marginality states that a player's payoff has to be calculated from her marginal contributions. Although this is well in line with

\footnotetext{
đAndré Casajus: Funded by the Deutsche Forschungsgemeinschaft (DFG, German Research Foundation) - 38839090. Frank Huettner: Funded by the Deutsche Forschungsgemeinschaft (DFG, German Research Foundation) - 288880950.

* Corresponding author

Email addresses: mail@frankhuettner.de (Frank Huettner), mail@casajus.de (André Casajus)

URL: www.frankhuettner.de (Frank Huettner), www. casajus.de (André Casajus)
} 
the economic tradition - e.g. Milnor (1952) argued that a reasonable outcome should be bounded by her maximal and by her minimal marginal contributions - it was Shapley (1953) who justified the marginality principle by showing that the basic axioms of Anonymity, Efficiency, additivity, and null player lead to a unique solution that assigns to each player her average marginal contribution in coalitions. Young (1985) established the reverse: when Anonymity, Efficiency, and marginality are required, the only possible solution is the Shapley value. This is often seen as a justification of the Shapley value, even though it might be puzzling as to why a player's merits in a game should solely be reflected by her marginal contributions. Indeed, the idea that player $i$ should simply get her marginal contribution within the grand coalition $v(N)-v(N \backslash i)$ is typically rejected based on the argument that this contribution is created jointly with the others. Hence, this contribution also reflects the productivity of the other players. But why can we ignore this marginal contribution when evaluating the other players, say $j \in N \backslash i$, as is required by the marginality principle?

A justification of the marginality principle derives from Harsanyi (1959), who developed the concept of dividends. Such a dividend $\eta(S)$ identifies the actual surplus of the particular combination of the players in this coalition $S$ by means of subtracting the dividends of all proper subcoalitions from the worth of this coalition, $\eta(S) \equiv v(S)-\sum_{T \subsetneq S} \eta(T)$. For example, in a game with two players, 1 and 2, the worth created by these two players $v(1,2)$ also encompasses their individual productivity, $\eta(1)=v(1)$ and $\eta(2)=v(2)$, so that their joint dividend is obtained as $\eta(1,2)=v(1,2)-v(1)-v(2)$. Note that the marginal contribution of player $i$ within $S$ exactly equals the dividends that become possible when she joins $S, v(S)-v(S \backslash i)=\sum_{T \subsetneq S: T \ni i} \eta(T)$. In this sense, the marginal contribution reflects the surplus creation that player $i$ is involved with. It further turns out that player $i$ 's marginal contributions, $\{v(S)-v(S \backslash i)\}_{S \subseteq N: S \ni i}$, determine the dividends that player $i$ is helping to create $\{\eta(S)\}_{S \subset N: S \ni i}$. The independence character of Marginality can therefore be justified by the equivalent axiom: a player's payoff shall not depend on surplus created by coalitions without this player, i.e., a change in dividend $\eta(T)$ has no impact on player $i$ 's payoff if $i \notin T$.

In this paper, we generalize these ideas to games with externalities and establish a characterization result analog to Young's. In the presence of externalities, the worth of a coalition $w(S, \pi)$ depends on coalitions formed by the other players, i.e., it depends on the partition $\pi \in \Pi(N \backslash S)$, where $\Pi(N \backslash S)$ is the set of all partitions of $N \backslash S$ (Lucas and Thrall, 1963). In order to express a player's marginal contribution within a coalition, we now have to clarify what to use as the worth of the coalition after the player has left, i.e., what to use as an analog to $v(S \backslash i)$. de Clippel and Serrano (2008) argue that a player could join any of the coalitions formed by the other players or stay alone and that these changes are the marginal contributions of this player within a coalition. The associated "Weak Marginality" property requires that if these changes remain the same for all coalitions to which a player belongs to, then this player's payoff should not change:

$$
\left.\begin{array}{l}
w(S, \pi)-w\left(S \backslash i, \pi_{+i \rightsquigarrow T}\right)=\tilde{w}(S, \pi)-\tilde{w}\left(S \backslash i, \pi_{+i \rightsquigarrow T}\right) \\
\text { for all } S \subseteq N: S \ni i \text {, all } \pi \in \Pi(N \backslash S), \text { and all } T \in \pi \cup \emptyset
\end{array}\right\} \Rightarrow \varphi_{i}(w)=\varphi_{i}(\tilde{w}),
$$

where $\pi_{+i \rightsquigarrow T} \in \Pi((N \backslash S) \cup i)$ denotes the partition obtained from $\pi \in \Pi(N \backslash S)$ when 
player $i \in S$ is added to component $T \in \pi \cup \emptyset$, with $\pi_{+i \rightsquigarrow \emptyset}$ indicating that player $i$ is added as a singleton component. They find that this axiom is rather weak and does not single out a solution concept together with Efficiency and Anonymity. We will argue that "Weak Marginality" might even be too strong since it ignores the possibility that the players outside of $S$ regroup when player $i \in S$ leaves.

We suggest another notion of marginality that will be in harmony with a generalization of Harsanyi's dividends. The key is to understand that in a game without externalities, the worth of a coalition when a player is removed can be seen as the worth that results when player $i$ was removed from the entire game, i.e., $v(S \backslash i)=v_{-i}(S \backslash i)$, where $v_{-i}$ denotes the subgame of $v$ that results when player $i$ is removed. We define the marginal contribution of player $i$ within embedded coalition $(S, \pi)$ as the change that occurs to its worth when player $i$ is removed from the game, i.e., $w(S, \pi)-w_{-i}^{r}(S \backslash i, \pi)$, where $w_{-i}^{r}$ denotes the subgame of $w$ when player $i$ is removed. At this point, we need to clarify how to derive a subgame when there are externalities. To this end, we use the concept of removal operators, which is a generalization of so-called restriction operators introduced by Dutta et al. (2010). A removal operator $r$ specifies how a subgame is derived from an original game, resorting to the information $w(S \backslash i, \hat{\pi}), \hat{\pi} \in \Pi((N \backslash S) \cup i)$ in order to determine $w_{-i}^{r}(S \backslash i, \pi)$. Given a path independent removal operator, i.e., if it does not matter in which order the players are removed, the definition of dividends becomes straight-forward, $\eta_{S, \pi}^{r}(w) \equiv w(S, \pi)-\sum_{T \subsetneq S} \eta_{T, \pi}^{r}\left(w_{-S \backslash T}^{r}\right)$.

We first identify those removal operators that allow us to establish "meaningful" notions of marginality. A removal operator $r$ must be linear in its components and path independent in order to guarantee that what we learn from a player's marginal contributions, $\{w(S, \pi)-$ $\left.w_{-i}^{r}(S \backslash i, \pi)\right\}_{S \subseteq N: S \ni i}$, is just the same as what we learn from the collection of dividends that this player is helping to create $\left\{\eta_{S, \pi}^{r}(w)\right\}_{S \subseteq N: S \ni i, \pi \in \Pi(N \backslash S)}$.

Our main result then shows that if such a removal operator $r$ is also anonymous, the corresponding $r$-Marginality, i.e.,

$$
\left.\begin{array}{l}
w(S, \pi)-w_{-i}^{r}(S \backslash i, \pi)=\tilde{w}(S, \pi)-\tilde{w}_{-i}^{r}(S \backslash i, \pi) \\
\text { for all } S \subseteq N: S \ni i, \text { all } \pi \in \Pi(N \backslash S)
\end{array}\right\} \quad \Rightarrow \quad \varphi_{i}(w)=\varphi_{i}(\tilde{w})
$$

together with Efficiency and Anonymity is characteristic of the $r$-Shapley value. This $r$ Shapley value is defined as the Shapley value applied to an auxiliary game with no externalities $v_{w}^{r}$ that is obtained from removing the player from the grand coalition, i.e., $v_{w}^{r}(S) \equiv w_{-N \backslash S}^{r}(S, \emptyset)$ for all $S \subseteq N$.

At first glance, it seems overly simplifying that all the information needed to compute the solution for the game $w$ should be found in $\left\{w_{-N \backslash S}^{r}(S, \emptyset)\right\}_{S \subseteq N}$. But a closer inspection reveals that these numbers contain condensed information about the whole game, in particular about the worths generated when $\pi \neq \emptyset$. If, however, the removal operator discards information about the original game, then so does the $r$-Shapley value. This insight that some information is ignored is already found and highlighted by de Clippel and Serrano (2008) for the specific removal operator $w_{-i}^{\text {singleton }}(S \backslash i, \pi) \equiv w(S \backslash i, \pi \cup i)$, which says that removing a player must be evaluated as if this player would stay alone. Then, the auxiliary 
game only refers to embedded coalitions $(S, \pi)$ for which $\pi$ is atomistic, and the $r$-Shapley value becomes the "externality free" Shapely value.

Although our axioms do not impose linearity on the solution directly, it is implicitly required through the properties that must be placed on the removal operator. Nonetheless, such removal operators might come in a natural formulation, making the resulting $r$-Marginality quite plausible. We illustrate this with the solution suggested by MachoStadler et al. (2007) for which we provide a new characterization. Apart from that solution, our approach allows for a characterization à la Young of virtually all generalizations of the Shapley value put forth in the literature and aides their understanding. In particular, we are able to identify the marginality principle characteristic of some solutions that are incompatible with the definition of "Weak Marginality" suggested by de Clippel and Serrano (2008). Finally, the introduced method also allows us to investigate egalitarian solutions and reveals how accounting for externalities may result in a deviation from the Shapley value. This is exemplified with a new solution that we introduce in order to determine payoffs if external effects are not supposed to be rewarded, while at the same time it cannot be assumed that the atomistic partition (or any other partition) is the default partition, i.e., there is no partition in which no externalities are exercised.

\section{The Shapley value, Marginality, and Independence of Alien Surplus}

Before we present our main results, we first review Young's characterization of the Shapley value and clarify the independence character of Marginality mentioned in the introduction. Let $\mathcal{N}$ contain all possible player sets and coalitions; i.e., $\mathcal{N}$ denotes the set of all finite subsets of the countably infinite universe of players. For $Q, S, T, N \in \mathcal{N}$, let $q, s, t$, and $n$ denote their cardinalities, respectively. For a given player set $N \in \mathcal{N}$, the worth $w(S, \pi)$ of a coalition $S \subseteq N$ depends on the coalitions formed by the other players, i.e., it depends on $\pi \in \Pi(N \backslash S)$, where $\Pi(N \backslash S)$ is the set of all partitions of $N \backslash S$ (for $S=N$, we denote the partition of the empty set by $\emptyset)$. A transferable utility game with externalities $w$, henceforth TUX game (also known as game in partition function form) assigns a worth to each embedded coalition $(S, \pi) \in \mathcal{E}(N)=\{(S, \pi) \mid S \subsetneq N$ and $\pi \in \Pi(N \backslash S)\} \cup\{N, \emptyset\}$, i.e., $w \in \mathbb{W}(N) \equiv\{f: \mathcal{E}(N) \rightarrow \mathbb{R} \mid f(\emptyset, \pi)=0$ for all $\pi \in \Pi(N)\}$; the set of all TUX games is denoted by $\mathbb{W} \equiv \bigcup_{N \in \mathcal{N}} \mathbb{W}(N)$.

If their are no externalities, i.e., if $w(S, \pi)=w(S, \tilde{\pi})$ for all $S \subseteq N$ and all $\pi, \tilde{\pi} \in$ $\Pi(N \backslash S)$, then the game is a called TU game (also known as a game in characteristic function) and we omit the partition when referring to the worth of a coalition. We denote the subspace of TU games by $\mathbb{V}(N) \equiv\left\{f: 2^{N} \rightarrow \mathbb{R} \mid f(\emptyset)=0\right\} \subseteq \mathbb{W}(N)$; let $\mathbb{V} \subseteq \mathbb{W}$ denote the collection of all TU games, $\mathbb{V} \equiv \bigcup_{N \in \mathcal{N}} \mathbb{V}(N)$.

A solution is a mapping $\varphi$ that assigns a payoff vector $\varphi(w) \in \mathbb{R}^{N}$ to any $N \in \mathcal{N}$ and $w \in \mathbb{W}(N)$. The Shapley value (Shapley, 1953), Sh, is defined for TU games and is given by

$$
\operatorname{Sh}_{i}(v) \equiv \sum_{S \subseteq N: S \ni i} \frac{(s-1) !(n-s) !}{n !}(v(S)-v(S \backslash i))
$$


for all $N \in \mathcal{N}, v \in \mathbb{V}(N)$, and $i \in N$. The Shapley value satisfies the following standard properties for solution concepts, which state that the name of a player does not matter and that the worth of the grand coalition is to be distributed.

Anonymity, A. For all $N \in \mathcal{N}, w \in \mathbb{W}(N)$, and permutations $\sigma$ of $N$, we have $\varphi(\sigma(w))=$ $\sigma(\varphi(w))$, where $\sigma(w)(S, \pi)=w(\sigma(S),\{\sigma(T) \mid T \in \pi\})$ for each embedded coalition $(S, \pi)$ and $\sigma(x)_{i}=x_{\sigma(i)}$ for each $x \in \mathbb{R}$ and each $i \in N$.

Efficiency, E. For all $N \in \mathcal{N}$ and $w \in \mathbb{W}(N)$, we have $\sum_{i \in N} \varphi_{i}(v)=w(N, \emptyset)$.

Since we can ignore the other players for the evaluation of the worth of a coalition $S$ in a TU game $v$, it is helpful to refer to the subgame in which those players are removed. For $v \in \mathbb{V}(N)$ and $T \subseteq N$, the subgame $v_{-T} \in \mathbb{V}(N \backslash T)$ is given by $v_{-T}(S)=v(S)$ for all $S \subseteq N \backslash T$. The Shapley value can be computed referring only to marginal contributions of a player, i.e., it satisfies the following property.

Marginality. For all $N \in \mathcal{N}, v, \tilde{v} \in \mathbb{V}(N)$, and $i \in N$, we have:

$$
\left.\begin{array}{l}
v(S)-v_{-i}(S \backslash i)=\tilde{v}(S)-\tilde{v}_{-i}(S \backslash i) \\
\text { for all } S \subseteq N: S \ni i
\end{array}\right\} \Rightarrow \varphi_{i}(v)=\varphi_{i}(\tilde{v})
$$

It was not clear until Young (1985) whether solutions that solely depend on a player's marginal contributions could differ from the Shapley value.

Theorem 1 (Young, 1985). The Shapley value is the unique solution for TU games that satisfies Efficiency, Anonymity, and Marginality.

To gain a better intuition for this, it is helpful to study dividends (Harsanyi, 1959), given by $\eta_{T}(v) \equiv v_{-N \backslash T}(T)-\sum_{S \subsetneq T} \eta_{N \backslash S}\left(v_{-N \backslash T}\right)$. Here, $\eta_{T}(v)$ identifies the surplus that is created by the very combination of the players in coalition $T$, as being the worth generated by this coalition reduced by the surplus that is already created by subcoalitions. It is well known that the Shapley value can also be computed from these dividends, $\mathrm{Sh}_{i}(v)=$ $\sum_{T \subseteq N: T \ni i} \eta_{T}(v) / t$. Note that each player's payoff only depends on the dividends of those coalitions, to which this player belongs to. In other words, the surplus (i.e., dividends) created without a player has no influence on this player's payoff. Thus, the Shapley value satisfies the following independence property.

Independence of Alien Surplus. For all $N \in \mathcal{N}, v, \tilde{v} \in \mathbb{V}(N)$, and $i \in N$, the following implication holds:

$$
\eta_{T}(v)=\eta_{T}(\tilde{v}) \text { for all } T \subseteq N: T \ni i \quad \Rightarrow \quad \varphi_{i}(v)=\varphi_{i}(\tilde{v}) .
$$

Young (1985) noticed that Marginality "is a type of independence condition". Indeed, Marginality is equivalent to the independence property above. Since $v(S)-v_{-i}(S \backslash i)=$ $\sum_{T \subseteq S: T \ni i} \eta_{T}(v)$, it is clear, that whenever the dividends $\{\eta(T)\}_{T \subseteq N: T \ni i}$ remain the same, then the marginal contributions remain the same. On the other hand, one easily shows by 
induction that whenever a player's marginal contributions stay the same, then the dividends stay the same. Thus, the hypotheses of Marginality and Independence of Alien Surplus are equivalent and so are the axioms.

Corollary 2. The Shapley value is the unique solution for TU games that satisfies Efficiency, Anonymity, and Independence of Alien Surplus.

\section{Player removal, Marginality, and independence in games with externalities}

When $i \in S$ leaves the embedded coalition $(S, \pi)$, it is unclear what partition of the other players with $i$ should be taken as a reference to define $i$ 's marginal contribution in $(S, \pi)$. To this end, we study removal operators. A removal operator is a mapping $r: \mathbb{W} \rightarrow \mathbb{W}$ that specifies for each game $w \in \mathbb{W}(N)$ and $i \in N$ a "subgame" $w_{-i}^{r} \in \mathbb{W}(N \backslash i)$. Given a removal operator $r$, we can then define the marginal contribution of $i$ within $S$ as

$$
w(S, \pi)-w_{-i}^{r}(S \backslash i, \pi) .
$$

Following Dutta et al. (2010) in their definition of restriction operators, we let the worth of $w_{-i}^{r}(S \backslash i, \pi)$ depend only on the worths that $S \backslash i$ can achieve in the original game, i.e., $w_{-i}^{r}(S \backslash i, \pi)$ depends on $w(S \backslash i, \hat{\pi}), \hat{\pi} \in \Pi((N \backslash S) \cup i)$. We deviate by making no assumption on the coalitions formed by the players in $(N \backslash S) \cup i$. This is different for restriction operators. Let $\pi_{+i \rightsquigarrow T} \in \Pi((N \backslash S) \cup i)$ denote the partition obtained from $\pi \in$ $\Pi(N \backslash S)$ when $i \in S$ is added to component $T \in \pi \cup \emptyset$, where $\pi_{+i \rightsquigarrow \emptyset}$ means that $i$ is added as a singleton component,

$$
\pi_{+i \rightsquigarrow T} \equiv \begin{cases}(\pi \backslash\{T\}) \cup\{T \cup i\}, & \text { if } T \in \pi \\ \pi \cup\{i\}, & \text { if } T \notin \pi\end{cases}
$$

A restriction operator as defined by Dutta et al. $(2010)$ is a removal operator with the property that it relies only on the information $w\left(S \backslash i, \pi_{+i \rightsquigarrow T}\right), T \in \pi \cup \emptyset$. For example, in a game with $N=\{1,2,3,4\}$, a restriction operator determines the worth $w_{-1}^{r}(2,3 \mid 4)$ based on $w(2,1|3| 4), w(2,13 \mid 4)$, and $w(2,14 \mid 3)$. A removal operator may also refer to $w(2,1 \mid 34)$ and $w(2,134)$.

For every removal operator $r$, we can now define a notion of $r$-Marginality for games with externalities.

$r$-Marginality, $\mathbf{M}^{r}$. For all $N \in \mathcal{N}, w, \tilde{w} \in \mathbb{W}(N)$, and $i \in N$, we have:

$$
\left.\begin{array}{l}
w(S, \pi)-w_{-i}^{r}(S \backslash i, \pi)=\tilde{w}(S, \pi)-\tilde{w}_{-i}^{r}(S \backslash i, \pi) \\
\text { for all } S \subseteq N: S \ni i, \text { all } \pi \in \Pi(N \backslash S)
\end{array}\right\} \quad \Rightarrow \quad \varphi_{i}(w)=\varphi_{i}(\tilde{w})
$$

$r$-Marginality requires that if player $i$ 's marginal contributions as specified by the removal operator $r$ are the same in two games, then this player's payoff should not change.

We further introduce a properties for removal operators. A removal operator is path independent if the order in which the players are removed is irrelevant, i.e., $\left(w_{-i}^{r}\right)_{-j}^{r}=$ 
$\left(w_{-j}^{r}\right)_{-i}^{r}$ for all $N \in \mathcal{N}, w \in \mathbb{W}(N)$, and $i, j \in N$. When $r$ is a path independent removal operator, we can refer to subgames where multiple players were removed, e.g. $w_{-S \backslash T}^{r} \in$ $\mathbb{W}(N \backslash T)$ for $T \subseteq S$ and $(S, \pi) \in \mathcal{E}(N)$. A removal operator is anonymous if it is neutral to player names, i.e., if $\sigma\left(w_{-i}^{r}\right)=(\sigma(w))_{-\sigma i}^{r}$. Finally, we say a removal operator $r$ is linear if $w_{-i}^{r}$ is linear in its components, i.e., there are linear mappings $f_{i, \hat{\pi}}^{r}$ such that

$$
w_{-i}^{r}(S \backslash i, \pi)=\sum_{\hat{\pi} \in \Pi((N \backslash S) \cup i)} f_{i, \hat{\pi}}^{r}(w(S \backslash i, \hat{\pi})) .
$$

Our insights rest on augmenting the insights on the relationship between marginal contributions and Harsanyi dividends of TU games in the domain of TUX games. For path independent removal operators, we can now introduce $r$-dividends $\eta_{S, \pi}^{r}(w)$ for TUX games. These dividends generalize the dividends for TU games and share some important conceptual and structural similarities. For all $N \in \mathcal{N}, w \in \mathbb{W}(N)$, and $(S, \pi) \in \mathcal{E}(N)$, we define $\eta_{S, \pi}^{r}(w)$ recursively by $\eta_{\emptyset, \pi}^{r}\left(w_{-S}\right)=0$ and

$$
\eta_{S, \pi}^{r}(w) \equiv w(S, \pi)-\sum_{T \subsetneq S} \eta_{T, \pi}^{r}\left(w_{-S \backslash T}^{r}\right)
$$

The dividend $\eta_{S, \pi}^{r}(w)$ identifies the surplus that is due to $(S, \pi)$ in $w$ by removing the surplus already created by subcoalitions $T \subseteq S$ when the players $S \backslash T$ would not be there (note that $\pi$ stays fixed).

The notion of $r$-dividends suggest an independence property that requires a player's payoff to depend solely on the surplus created with this player.

$r$-Independence of Alien Surplus, $\mathbf{I}^{r}$. For all $N \in \mathcal{N}, w, \tilde{w} \in \mathbb{W}(N)$, and $i \in N$, we have:

$$
\left.\begin{array}{l}
\eta_{S, \pi}^{r}(w)=\eta_{S, \pi}^{r}(\tilde{w}) \\
\text { for all } S \subseteq N: S \ni i, \text { all } \pi \in \Pi(N \backslash S)
\end{array}\right\} \Rightarrow \varphi_{i}(w)=\varphi_{i}(\tilde{w})
$$

Marginality and Independence of Alien Surplus are equivalent if there are no externalities. We can preserve this equivalence for specific removal operators.

Proposition 3. Let $r$ be a path independent and linear removal operator. For all $N \in \mathcal{N}$, $w, \tilde{w} \in \mathbb{W}(N)$, and $i \in N$, the following statements are equivalent:

(i) $w(S, \pi)-\tilde{w}_{-i}^{r}(S \backslash i, \pi)=w(S, \pi)-\tilde{w}_{-i}^{r}(S \backslash i, \pi)$ for all $(S, \pi) \in \mathcal{E}(N)$ such that $S \ni i$.

(ii) $\eta_{T, \pi}^{r}\left(w_{-S \backslash T}^{r}\right)=\eta_{T, \pi}^{r}\left(\tilde{w}_{-S \backslash T}^{r}\right)$ for all $(S, \pi) \in \mathcal{E}(N)$ and $T \subseteq S$ such that $T \ni i$.

The details of the proof are referred to the appendix. In the next section, we provide a characterizations using this insight. 


\section{Independence (or Marginality), Efficiency, and Anonymity are characteristic of the $r$-Shapley value}

Given a path independent removal operator $r$ and TUX game $w \in \mathbb{W}(N)$, we can derive the TU game $v_{w}^{r} \in \mathbb{V}(N)$, given by

$$
v_{w}^{r}(S)=w_{-N \backslash S}^{r}(S, \emptyset)
$$

for $S \subseteq N$. This TU game captures a lot of information of the original TUX game and applying the Shapley value to this game yields a natural extension of the Shapely value to TUX games. The $r$-Shapley value, $\mathrm{Sh}^{r}$, is defined as

$$
\operatorname{Sh}^{r}(w)=\operatorname{Sh}\left(v_{w}^{r}\right)
$$

for all $N \in \mathcal{N}$ and $w \in \mathbb{W}(N)$. It is exactly this solution, that is characterized by Efficiency, Anonymity, and $r$-Independence of Alien Surplus or $r$-Marginality.

Theorem 4. Let $r$ be a path independent, linear, and anonymous removal operator. Then, the $r$-Shapley, $\mathrm{Sh}^{r}$, value is the unique solution that satisfies Efficiency $(\boldsymbol{E})$, Anonymity (A), and (r-Independence of Alien Surplus $\left(\boldsymbol{I}^{r}\right)$ or $r$-Marginality $\left.\left(\boldsymbol{M}^{r}\right)\right)$.

In the remainder of this section, we sketch the proof (some details are referred to the appendix). A discussion of the Theorem and its applications are given in the next section.

Since $\eta_{S, \emptyset}^{r}\left(w_{-N \backslash S}^{r}\right)=\eta_{S}\left(v_{w}^{r}\right), \eta^{r}$ is an extension of the Harsanyi dividends of the TU game $v_{w}^{r}$ to the TUX game $w$, and these dividends can be used to compute the $r$-Shapley value,

$$
\operatorname{Sh}^{r}(w)=\sum_{S \subseteq N: S \ni i} \frac{\eta_{S, \emptyset}^{r}\left(w_{-N \backslash S}^{r}\right)}{s} .
$$

Proposition 3 and (5) guarantee that $\mathrm{Sh}^{r}$ satisfies $\mathbf{M}^{r}$. For the uniqueness result, we employ a technique from Moulin (1988) and Pintér (2015). In contrast to the proof by Young (1985), we do not need a basis (of unanimity games) of the vector space $\mathbb{W}(N)$. The proof is by induction on the number of symmetric players in $w$. Suppose $\varphi$ satisfies $\mathbf{A}, \mathbf{I}^{r}$, and $\mathbf{E}$. If all players are symmetric, $\mathbf{A}$ and $\mathbf{E}$ determine the payoff $\varphi_{j}(w)=w(N, \emptyset) / n=\operatorname{Sh}_{j}^{r}(w)$. To understand the induction step, suppose there are $n-1$ symmetric players in $w$ and let $\hat{\imath}$ be the player not symmetric to the others. We can obtain a modified game $\tilde{w}$ from $w$ by changing the dividends of the symmetric players so that they equal the dividends of $\hat{\imath}$, while keeping the dividends of $\hat{\imath}$ in $w$ and $\tilde{w}$ unchanged. This way, all the $n$ players are symmetric in $\tilde{w}$ and we know that payoffs in $\tilde{w}$ are given by the induction hypothesis, $\varphi_{i}(\tilde{w})=\operatorname{Sh}_{i}^{r}(\tilde{w})$. Using $\mathbf{I}^{r}$, we further determine the payoff of $\hat{\imath}$ in $w, \varphi_{\hat{\imath}}(w)=\operatorname{Sh}_{\hat{\imath}}^{r}(\tilde{w})$. This way, we learn all payoffs in $w$ except for the payoffs of the symmetric players. $\mathbf{E}$ determines what they can get, $\sum_{j \neq \hat{\imath}} \varphi_{j}(w)=w(N, \emptyset)-\varphi_{\hat{\imath}}(w)=\sum_{j \neq \hat{\imath}} \operatorname{Sh}_{j}^{r}(w)$ and $\mathbf{A}$ guarantees that $\varphi$ shares this equally among them as does $\operatorname{Sh}^{r}, \varphi_{j}(w)=\sum_{j \neq \imath} \operatorname{Sh}_{j}^{r}(w) /(n-1)=\operatorname{Sh}_{j}^{r}(w)$ for $j \neq \hat{\imath}$. 


\section{Discussion and application}

In face of Young's result and since the $r$-Shapley value is the Shapley value of $v_{w}^{r}$, it perhaps appears less surprising at first glance that Efficiency, Symmetry, and $r$-Independence of Alien Surplus are sufficient to single out a solution. However, the assumption that corresponds to Independence of Alien Surplus for $v_{w}^{r}$ would be a much stronger assumption than $r$-Independence of Alien Surplus, namely:

$$
\eta_{S, \emptyset}^{r}\left(w_{-N \backslash S}^{r}\right)=\eta_{S, \emptyset}^{r}\left(\tilde{w}_{-N \backslash S}^{r}\right) \text { for all } S \subseteq N: S \ni i \Rightarrow \varphi_{i}(w)=\varphi_{i}(\tilde{w}) .
$$

In fact, replacing $r$-Independence of Alien Surplus with assumption (6) in Theorem 4 would be a straight-forward extension of Young's result. To see that (6) is a stronger than $r$ Independence of Alien Surplus, note that (6) does not condition on $\eta_{T, \pi}^{r}\left(w_{-S \backslash T}^{r}\right)$ for $\pi \neq \emptyset$. In other words, in the presence of Efficiency and Symmetry, the information that is stored in these numbers is discarded for the determination of a player's payoff.

Depending on the removal operator, (6) could become a very simple and strong statement. For example, for the removal operator $w_{-i}^{\text {singleton }}(S \backslash i, \pi) \equiv w(S \backslash i, \pi \cup i)$, which says that removing a player must be evaluated as if this player would stay alone, the auxiliary game only refers to embedded coalitions $(S, \pi)$ for which $\pi$ is atomistic, $v_{w}^{r}(S)=$ $w(S,\{j \mid j \in N \backslash S\})$. Then, (6) reads as follows:

$$
\left.\begin{array}{l}
w(S,[N \backslash S])-w(S \backslash i,[N \backslash S] \cup i)=\tilde{w}(S,[N \backslash S])-\tilde{w}(S \backslash i,[N \backslash S] \cup i) \\
\text { for all } S \subseteq N: S \ni i \text { and with }[N \backslash S]=\{j \mid j \in N \backslash S\}
\end{array}\right\} \Rightarrow \varphi_{i}(w)=\varphi_{i}(\tilde{w}) .
$$

This is a much stronger assumption than singleton-Marginality, which also conditions on $w(S, \pi)$ for nonatomistic $\pi$ :

$$
\left.\begin{array}{l}
w(S, \pi)-w(S \backslash i, \pi \cup i)=\tilde{w}(S, \pi)-\tilde{w}(S \backslash i, \pi \cup i) \\
\text { for all } S \subseteq N: S \ni i \text { and all } \pi \in \Pi(N \backslash S)
\end{array}\right\} \Rightarrow \varphi_{i}(w)=\varphi_{i}(\tilde{w})
$$

de Clippel and Serrano (2008) already notice and stress that in the presence of Efficiency and Anonymity, singleton-Marginality must be strenghened to (7). This can be stated more general. The $r$-Shapley value satisfies condition (6) and whenever the removal operator systematically ignores information of the game, (6) conditions on less information than $r$-Marginality, so that a player $i$ 's payoff depends less input.

Although our axioms do not impose linearity on the solution directly, it might be implicitly required through the assumptions that must be placed on the removal operator. Nonetheless, such removal operators might come in a natural formulation such that the averaging that is at work appears quite plausible. We first illustrate this for various generalizations offered in the literature. Thereafter, we show how deviations from the Shapley value can be motivated by externalities.

\subsection{Characterizations of Various Generalizations of the Shapley value}

Macho-Stadler et al. (2007) and Skibski et al. (2018) put forth a solution that emerges from a coalition formation procedure following the Chinese restaurant process. This process 
can be described as follows. Consider a "Chinese restaurant" with $n$ round tables each seating $n$ persons. The players arrive at the restaurant in some order. The first player takes a seat at one of the tables. Any following player takes seat at an empty table or joins any of the already present players with the same probability. Note that this way it is more likely that a player takes seat at a table with more players. This process induces an anonymous probability distribution over partitions. Concretely, after $n$ players have entered the room, each table is a component of $\pi \in \Pi(N)$ and $\pi$ has the probability $p(\pi) \equiv \prod_{T \in \pi}(t-1) ! / n$ !. Using this probability distribution, we can compute an average TU game $\bar{v}_{w}$ in which each coalitions gets the expected value of $w(S, \pi)$ over all partitions $\pi \in \Pi(N \backslash S)$, i.e., $\bar{v}_{w}(S) \equiv \sum_{\pi \in \Pi(N \backslash S)} p(\pi) w(S, \pi)$ for $S \subseteq N$. The average Shapley value $\mathrm{Sh}^{\star}$ is defined as the Shapley value of this TU game,

$$
\mathrm{Sh}^{\star}(w) \equiv \operatorname{Sh}\left(\bar{v}_{w}\right)
$$

for all $N \in \mathcal{N}, w \in \mathbb{W}(N)$.

Now, let $\pi(j)$ denote the component of $\pi \in \Pi(N \backslash S)$ that contains $j \in N \backslash S$. Then, $\pi_{+i \rightsquigarrow \pi(j)} \in \Pi((N \backslash S) \cup i)$ is the partition obtained from $\pi \in \Pi(N \backslash S)$ if player $i$ is added and joins the component of player $j$ in $\pi$, where $\pi_{+i \rightsquigarrow \pi(i)}$ means that $i$ forms a singleton component. Macho-Stadler et al. (2007) observe that the average Shapley value satisfies the following marginality property.

$\star$-Marginality, $\mathbf{M}^{\star}$. For all $N \in \mathcal{N}, w, v \in \mathbb{W}(N)$, and $i \in N$, we have:

$w(S, \pi)-\frac{1}{n-s+1} \sum_{j \in(N \backslash S) \cup i} w\left(S \backslash i, \pi_{+i \rightsquigarrow \pi(j)}\right)=\tilde{w}(S, \pi)-\frac{1}{n-s+1} \sum_{j \in(N \backslash S) \cup i} \tilde{w}\left(S \backslash i, \pi_{+i \rightsquigarrow \pi(j)}\right)$

for all $S \subseteq N, S \ni i$, and all $\pi \in \Pi(N \backslash S)$ implies $\varphi_{i}(w)=\varphi_{i}(v)$.

Here, the marginal contribution of player $i$ within coalition $S$ is derived from the assumption that when player $i$ leaves $S$, player $i$ may equally likely be attracted by every player $j$ outside of $S$ or by himself, and joins into player $j$ 's coalition or player $i$ stays alone. Thus, *-Marginality does not directly impose an average over partitions but stipulates that the players are equally likely teaming up with other individuals (and their peers). This is particularly plausible if $\pi$ represents the clusters of a network and player $i$ is expected to form new links to players in $N \backslash S$ after cutting ties to the players in $S$.

Obviously, $\star$-Marginality is induced by the $\star$-removal operator given by $w_{-i}^{\star}(S \backslash i, \pi)=$ $\frac{1}{n-s+1} \sum_{j \in(N \backslash S) \cup i} w\left(S \backslash i, \pi_{+i \rightsquigarrow \pi(j)}\right)$ for all $(S \backslash i, \pi) \in \mathcal{E}(N \backslash i)$. From Theorem 4 , we obtain a new characterization of the average Shapley value.

Corollary 5. The average Shapley value, $\mathrm{Sh}^{\star}$, is the unique solution satisfying Efficiency $(\boldsymbol{E})$, Anonymity $(\boldsymbol{A})$, and $\star$-Marginality $\left(\boldsymbol{M}^{\star}\right)$.

Again, it is helpful to have a look at condition (6) for the $\star$-removal operator. To this end, we examplarily determine $w_{-N \backslash S}^{\star}(S, \emptyset)$ for a game with for players, $N=\{1,2,3,4\}$. We note 
that $w_{-1}^{\star}(234, \emptyset)=w(234,1)$ and $w_{-12}^{\star}(34, \emptyset)=w_{-2}^{\star}(234,1)=\frac{1}{2} w(34,12)+\frac{1}{2} w(34,1 \mid 2)$. By the same logic, we recursively obtain

$$
\begin{aligned}
w_{-123}^{\star}(4, \emptyset) & =w_{-12}^{\star}(4,3) \\
& =\frac{w_{-1}^{\star}(4,23)+w_{-1}^{\star}(4,2 \mid 3)}{2} \\
& =\frac{\frac{w(4,213)+w(4,231)+w(4,1 \mid 23)}{3}+\frac{w(4,12 \mid 3)+w(4,2 \mid 13)+w(4,1|2| 3)}{3}}{2} \\
& =\sum_{\pi \in \Pi(\{1,2,3\})} p(\pi) w(4, \pi)
\end{aligned}
$$

Perhaps not surprisingly, one can show that the auxiliary game is just the average game,

$$
v_{w}^{\star}(S)=w_{-N \backslash S}^{\star}(S, \emptyset)=\sum_{\pi \in \Pi(N \backslash S)} p(\pi) w(S, \pi)=\bar{v}_{w}(S) .
$$

Thus, condition (6) just reads

$$
\left.\begin{array}{l}
\bar{v}_{w}(S)-\bar{v}_{w}(S \backslash i)=\bar{v}_{\tilde{w}}(S)-\bar{v}_{\tilde{w}}(S \backslash i) \\
\text { for all } S \subseteq N: S \ni i
\end{array}\right\} \Rightarrow \varphi_{i}(w)=\varphi_{i}(\tilde{w})
$$

According to (8) player $i$ 's performance is measured in terms of marginal contributions to the average worth of a coalition. In contrast to this, $\star$-Marginality states that player $i$ 's performance is measured in terms of marginal contributions in a coalition where the worth of a coalition without player $i$ 's is the average over various coalitions.

Further characterizations can be derived from Theorem 4. The power of these characterizations lies in the fact that each marginality property reveals how externalities are aggregated to a measure of the merits of a player in a game. In particular, a player whose marginal contributions are zero is considered a null player. For example, a $\star$-Null player is a player who when reaching out to players outside her embedded coalition is expected to have no impact on the worth of this embedded coalition. In chronological order, we list further concepts studied in the literature:

1. Using the removal operator, $w_{-i}^{\text {stable average }}(S \backslash i, \pi)=\frac{1}{|\pi|+1} \sum_{T \in \pi \cup \emptyset} w\left(S \backslash i, \pi_{+i \rightsquigarrow T}\right)$, we obtain the marginality property characteristic of the solution introduced by Bolger (1989);

2. Using the removal operator, $w_{-i}^{\text {regroup switch }}(S \backslash i, \pi)=\frac{|\Pi(N \backslash S)|}{|\Pi((N \backslash S) \cup i)|} \sum_{T \in \pi \cup \emptyset} w\left(S \backslash i, \pi_{+i \rightsquigarrow T}\right)$, we obtain the marginality property characteristic of the solution introduced by Albizuri et al. (2005);

3. Using the singleton removal operator, $w_{-i}^{\text {singleton }}(S \backslash i, \pi)=w(S \backslash i, \pi \cup i)$, we obtain the "Marginality" property and the characterization result of de Clippel and Serrano (2008, Proposition 3), which is an axiomatization of the "externality free value" introduced by Pham Do and Norde (2007); 
4. Using a linear combination of the average and the singleton removal operators, $w_{-i}^{\theta}(S \backslash i, \pi)=$ $w_{-i}^{\text {singleton }}(S \backslash i, \pi)+\theta w_{-i}^{\text {stable average }}(S \backslash i, \pi), \theta \geq 0$, we obtain the marginality property characteristic of the solutions selected by Dutta et al. (2010, Theorem 2);

5. Using the removal operator, $w_{-i}^{\text {altogether }}(S \backslash i, \pi)=w(S \backslash i,\{(N \backslash S) \cup i\})$, we obtain the marginality property characteristic of the solution put forth by McQuillin (2009);

6. Using the removal operator, $w_{-i}^{\text {regroup average }}(S \backslash i, \pi)=\frac{1}{|\Pi((N \backslash S) \cup i)|} \sum_{\hat{\pi} \in|\Pi((N \backslash S) \cup i)|} w(S \backslash i, \hat{\pi})$, we obtain the marginality property characteristic of the solution introduced by $\mathrm{Hu}$ and Yang (2010).

The solution 6 above does not satisfy "Weak Marginality" as defined in (1). To see why, recall that a removal operator can refer to the worth of $S \backslash i$ for all partitions $\hat{\pi} \in$ $\Pi((N \backslash S) \cup i)$. This is not the case for the removal operators in 1, 3, 4, 5 and in Corollary 5 for which the induced solutions satisfy "Weak Marginality". These removal operators make use of less information and only draw information from $w\left(S \backslash i, \pi_{+i \rightsquigarrow T}\right), T \in \pi \cup \emptyset$, i.e., these removal operators are restriction operators as studied in Dutta et al. (2010).

\subsection{Deviation from the Shapley value}

van den Brink (2007) has shown that the Shapley value and the equal division value given by

$$
\operatorname{ED}_{i}(w) \equiv \frac{w(N, \emptyset)}{n}
$$

for all $N \in \mathcal{N}, w \in \mathbb{W}(N)$, and $i \in N$, share similar characteristics for TU games. To see why, consider the following property that is entailed from the trivial removal operator $w_{-i}^{0}(S \backslash i, \pi)=0$.

0-Marginality, $\mathbf{M}^{0}$. For all $N \in \mathcal{N}, w, \tilde{w} \in \mathbb{W}(N)$, and $i \in N$, we have:

$$
w(S, \pi)=\tilde{w}(S, \pi) \text { for all } S \subseteq N: S \ni i \text {, and all } \pi \in \Pi(N \backslash S) \quad \Rightarrow \quad \varphi_{i}(w)=\varphi_{i}(\tilde{w})
$$

On the domain of TU games, 0-Marginality coincides with Coalitional Marginality discussed by van den Brink (2007). Obviously, Theorem 4 applies and we obtain a generalization of (van den Brink, 2007, Theorem 3.3) from TU games to TUX games.

Corollary 6. The equal division value, ED, is the unique solution satisfying Efficiency $(\boldsymbol{E})$, Anonymity $(\boldsymbol{A})$, and 0-Marginality $\left(\boldsymbol{M}^{0}\right)$.

The previous corollary indicates that accounting externalities can lead to a deviation from the Shapley value. This is a consequence of the fact that in a TUX game, it is hard to separate the productive value of a player from this player's external effect. Whereas de Clippel and Serrano (2008) suggest that deviating from singletons constitutes the creation of external effects, we may argue that if there is no "natural" partition of the players (in particular, if the atomistic partition is not viewed as the default), it is unclear what could be the actual productivity if externalities have to be discarded. We now introduce a solution for this purpose, i.e., a solution with the ambition to distribute $w(N, \emptyset)$ without rewarding external effects and without making an assumption about the natural partition of players. 
Consider the following partial order " $\leq$ " on the set of all embedded coalitions $\mathcal{E}$ :

$$
(T, \tau) \leq(S, \pi) \text { if } T \subseteq S \text { and } \tau_{-S \backslash T}=\pi,
$$

where $\tau_{-S \backslash T}$ is the partition obtained from $\tau$ when canceling the players in $S \backslash T, \tau_{-S \backslash T} \equiv$ $\{T \backslash S \mid T \in \tau\}$. If $(T, \tau) \leq(S, \pi)$, then the embedded coalition can mimic the configuration $(T, \tau)$ simply by sending the players in $T \backslash S$ to their blocks in $\pi$. The corresponding $\leq$ unanimity game

$$
u_{\bar{T}, \tau}^{\leq}(S, \pi) \equiv \begin{cases}1, & \text { if } T \subseteq S \text { and } \tau_{-S \backslash T}=\pi \\ 0, & \text { else. }\end{cases}
$$

has a natural interpretation: the players in coalition $T$ can generate $\$ 1$ if the others are grouped according to $\tau$ and there is no other value generation possible. Now, every embedded coalition that can mimic $(T, \tau)$ can also generate $\$ 1$. For example, suppose that some $j \notin T$ leaves his coalition $\tau(j) \in \tau$ and joins coalition $T$. Then it should still be possible to generate $\$ 1$, because the players from $T$ can agree with player $j$ that it is best that he goes out to his original coalition and works there. Accordingly, one could argue that player $j$ neither contributes nor destroys anything when moving around, and hence should receive zero payoff. The solution $\leq$-Shapley value does exactly this and gives $1 / t$ to each player in coalition $T$ in game $u_{T, \tau}^{\leq}$and zero to the others. In general, we define the $\leq$-Shapley value as

$$
\operatorname{Sh}_{i}^{\leq}(w) \equiv \sum_{(T, \tau) \in \mathcal{E}(N): T \ni i} \frac{m_{w}^{\leq}(T, \tau)}{t} .
$$

where $m_{w}^{\leq}(T, \tau)$ denotes the Möbius inverse given by

$$
m_{w}^{\leq}(T, \tau) \equiv w(T, \tau)-\sum_{(S, \pi) \npreceq(T, \tau)} m_{w}^{\leq}(S, \pi) .
$$

It transpires that $\mathrm{Sh}^{\leq}$is compatible with the removal operator given by

$$
w_{-i}^{\leq}(S \backslash i, \pi) \equiv \sum_{(T, \tau) \in \mathcal{E}(N):(T, \tau) \leq(S, \pi), T \subseteq S \backslash i} m_{w}^{\leq}(T, \tau),
$$

since $w(S, \pi)-w_{-i}^{\leq}(S \backslash i, \pi)=\tilde{w}(S, \pi)-\tilde{w}_{-i}^{\leq}(S \backslash i, \pi)$ for all $S \ni i$ and $\pi \in \Pi(N \backslash S)$ then indeed means that $w$ and $\tilde{w}$ have identical same Möbius inverse for all coalitions including $T$, i.e., $m_{w}^{\leq}(T, \tau)=m_{\tilde{w}}^{\leq}(T, \tau)$ for all $T \ni i$ and $\tau \in \Pi(N \backslash T)$. Interestingly, the removal operator above has a simple expression.

Lemma 7. For the $\leq$-removal operator, we have $w_{-i}^{\leq}(S \backslash i, \pi)=\sum_{T \in \pi \cup \emptyset} w\left(S \backslash i, \pi_{+i \rightsquigarrow T}\right)$.

This suggests the following notion of marginality:

$\leq$-Marginality, $\mathbf{M} \leq$. For all $N \in \mathcal{N}, w, \tilde{w} \in \mathbb{W}(N)$, and $i \in N$, we have:

$\left.\begin{array}{l}w(S, \pi)-\tilde{w}(S, \pi)=\sum_{T \in \pi \cup \emptyset}\left[w\left(S \backslash i, \pi_{+i \rightsquigarrow T}\right)-\tilde{w}\left(S \backslash i, \pi_{+i \rightsquigarrow T}\right)\right] \\ \text { for all } S \subseteq N, S \ni i, \text { and all } \pi \in \Pi(N \backslash S)\end{array}\right\} \Rightarrow \varphi_{i}(w)=\varphi_{i}(\tilde{w})$. 
Suppose each embedded coalition $(S, \pi)$ has the ability to deploy its players to any other block $T \in \pi \cup \emptyset$, resulting in the worth of $w\left(S \backslash i, \pi_{+i \rightsquigarrow T}\right)$. Aggregated over all target coalitions, this reflects the extend to which this player contributes only in the form of external effects $\sum_{T \in \pi \cup \emptyset} w\left(S \backslash i, \pi_{+i \rightsquigarrow T}\right)$. If a player's true value is not supposed to be measured by the extend to which this player can generate external effects on $(S, \pi)$, but instead if we are interested in rewarding a player according to this player's genuine creation of worth, then we have to discard these external effects. In particular, if the worth of a player's embedded coalitions only changes by the extend to which this player can exercise external effects, we can claim that the genuine productivity of this player did not change. According to $\leq-$ Marginality, the player's payoff should not change neither. From Theorem 4 and Lemma 7. we single out the $\leq$-Shapley value as the only solution that achieves this goal.

Theorem 8. The $\leq$-Shapley value, $\mathrm{Sh}^{\leq}$, is the unique solution satisfying Efficiency $(\boldsymbol{E})$, Symmetry $(\boldsymbol{A})$, and $\leq$-Marginality $(\boldsymbol{M} \leq)$.

The $\leq$-Shapley value does not coincide with the Shapley value if there are no externalities. In order to see this more lucidly, we determine the auxiliary game for a game with four players. Note that $w_{-1}^{\leq}(234, \emptyset)=w(234,1), w_{-12}^{\leq}(34, \emptyset)=w_{-2}^{\leq}(234,1)=w(34,12)+$ $w(234,1 \mid 2)$, and

$$
\begin{aligned}
w_{-123}^{\leq}(4, \emptyset) & =w_{-12}^{\leq}(4,3) \\
& =w_{-1}^{\leq}(4,23)+w_{-1}^{\leq}(4,2 \mid 3) \\
& =w(4,123)+w(4,1 \mid 23)+w(4,12 \mid 3)+w(4,13 \mid 2)+w(4,1|2| 3)
\end{aligned}
$$

In general, we have

$$
v_{w}^{\leq}(S)=w_{-N \backslash S}^{\leq}(S, \emptyset)=\sum_{\pi \in \Pi(N \backslash S)} w(S, \pi),
$$

so that applying the $\leq$-Shapley value to a game without externalities actually becomes

$$
\operatorname{Sh}_{i}^{\leq}(v)=\sum_{S \subseteq N: S \ni i} \frac{(s-1) !(n-s) !}{n !}\left(B_{n-s} v(S)-B_{n-s+1} v(S \backslash i)\right)
$$

where the $n^{\text {th }}$ Bell number $B_{n}=|\Pi(N)|$ is the number of partitions of a set with $n$ elements.

Note that $B_{n-s}-B_{n-s+1}$ is decreasing in $s$; e.g., the contribution in the grand coalition is fully rewarded $B_{n-n} v(N)-B_{n-n+1} v(N \backslash i)=v(N)-v(N \backslash i)$, while $B_{n-2} v(\{i, j\})-$ $B_{n-1} v(j) \approx B_{n-2} v(\{i, j\})$ for large $n$, i.e., the $\leq$-Shapley value roughly satisfies coalitional monotonicity on small coalitions and treats their impact similar to the equal division value (see Corollary 6). The intuition behind this is clear: the more players outside a coalition can influence a coalition's worth through external effects, the more difficult it becomes to attribute productivity to its source if external effects are not regarded as source of productivity.

From (14), we further see that whenever $w(S, \pi)=0$ for $S \neq N$ and nonatomistic partitions $\pi$, the $\leq$-Shapley value coincides with the "externality free" Shapley value $\mathrm{Sh}^{\text {singleton }}$. 
In other words, the "externality free" Shapley value is obtained from the $\leq$-Shapley value if the singleton partitions are declared to be the default and all other worths are set to zero, i.e., $\operatorname{Sh}^{\text {singleton }}(w)=\operatorname{Sh}^{\leq}(\dot{w})$ where $\dot{w}(S, \pi)=w(S, \pi)$ if $\pi$ is atomistic or $S=N$, and $\dot{w}(S, \pi)=0$ otherwise. This indicates once more that the $\leq$-Shapley value can be seen as the externality free Shapley value if there is no default partition.

\section{Appendix A. Computing $r$-dividends}

The following lemmas contain several properties of $r$-dividends that constitute the necessary ingredients to establish Proposition 3. These properties are familiar from dividends for TU games. First, $\eta_{S, \pi}^{r}(w)$ can be computed utilizing the inclusion-exclusion principle.

Lemma 9. Let $r$ be a path independent removal operator. For all $N \in \mathcal{N}, w \in \mathbb{W}(N)$, $(S, \pi) \in \mathcal{E}(N)$, we have

$$
\eta_{S, \pi}^{r}(w)=\sum_{T \subseteq S}(-1)^{s-t} w_{-S \backslash T}^{r}(T, \pi)
$$

Based on the previous lemma, we can relate the dividends $\eta^{r}$ with marginal contributions.

Lemma 10. Let $r$ be a path independent and additive removal operator. For all $N \in \mathcal{N}$, $(S, \pi) \in \mathcal{E}(N), w \in \mathbb{W}(N)$, and $i \in S$, we have

$$
w(S, \pi)-w_{-i}^{r}(S \backslash i, \pi)=\sum_{T \subseteq S: T \ni i} \eta_{T, \pi}^{r}\left(w_{-S \backslash T}^{r}\right) .
$$

\section{Appendix A.1. Proof of Lemma 9}

We show the first claim by induction on $|N|$. Induction basis: The claim is obvious from (3) for $n \leq 1$. Induction hypothesis (IH): Let Lemma 9 hold true for all $n \leq \ell$.

Induction step: Let $N \in \mathcal{N}$ by such that $n=\ell+1$ and $w \in \mathbb{W}(N)$. For $(S, \pi) \in \mathcal{E}(N)$, we obtain

$$
\begin{aligned}
\eta_{S, \pi}^{r}(w) & \stackrel{\sqrt[3]{=}}{=} w(S, \pi)-\sum_{T \subsetneq S} \eta_{T, \pi}^{r}\left(w_{-S \backslash T}^{r}\right) \\
& \stackrel{I H}{=} w(S, \pi)-\sum_{T \subsetneq S} \sum_{Q \subseteq T}(-1)^{t-q} w_{-S \backslash Q}^{r}(Q, \pi) \\
& =w(S, \pi)-\sum_{Q \subsetneq S} w_{-S \backslash Q}^{r}(Q, \pi) \sum_{T: Q \subseteq T \subsetneq S}(-1)^{t-q} .
\end{aligned}
$$

$\operatorname{Using} \sum_{T: Q \subseteq T \subsetneq S}(-1)^{t-q}=\sum_{t=q}^{s-1}(-1)^{t-q}\left(\begin{array}{c}s-q \\ t-q\end{array}\right)=\sum_{t=0}^{s-1-q}(-1)^{t}\left(\begin{array}{c}s-q \\ t\end{array}\right)=(-1)^{s-q+1}$ concludes the proof,

$$
\eta_{S, \pi}^{r}(w)=w(S, \pi)+\sum_{Q \subsetneq S}(-1)^{s-q} w_{-S \backslash Q}^{r}(Q, \pi) .
$$




\section{Appendix A.2. Proof of Lemma 10}

Fix $N \in \mathcal{N}, S, \pi, w \in \mathbb{W}(N)$, and $i \in S$. We show $w_{-i}^{r}(S \backslash i, \pi)=\sum_{Q \subseteq S \backslash i} \eta_{Q, \pi_{-S \backslash Q}}^{r}\left(w_{-S \backslash Q}^{r}\right)$. The claim then follows from (3). For any $\hat{\pi} \in \Pi((N \backslash S) \cup i)$, we have

$$
w_{-i}^{r}(S \backslash i, \hat{\pi}) \stackrel{\sqrt[3]{3}}{=} \sum_{Q \subseteq S \backslash i} \eta_{Q, \hat{\pi}}^{r}\left(w_{-(S \backslash i) \backslash Q}^{r}\right)
$$

and since $r$ is additive, we get

$$
w_{-i}^{r}(S \backslash i, \pi)=\sum_{Q \subseteq S \backslash i} f_{i, \hat{\pi}}^{r}\left(\left(\eta_{Q, \hat{\pi}}^{r}\left(w_{-(S \backslash i) \backslash Q}^{r}\right)\right)_{\hat{\pi} \in \Pi((N \backslash S) \cup i)}\right) .
$$

For all $Q \subseteq S \backslash i$ and all $\hat{\pi} \in \Pi((N \backslash S) \cup i)$, we further have

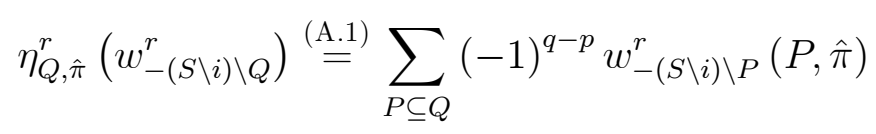

which inserted in A.3 and applying linearity of $r$ and of $\eta$ gives

$$
w_{-i}^{r}(S \backslash i, \pi)=\sum_{Q \subseteq S \backslash i} \sum_{P \subseteq Q}(-1)^{q-p} f_{i, \hat{\pi}}^{r}\left(\left(w_{-(S \backslash i) \backslash P}^{r}(P, \hat{\pi})\right)_{\hat{\pi} \in \Pi((N \backslash S) \cup i)}\right) .
$$

Note that $\left(w_{-S \backslash P}^{r}\right)(P, \pi)=\left(w_{-(S \backslash i) \backslash P}^{r}\right)_{-i}^{r}(P, \pi)=f_{i, \hat{\pi}}^{r}\left(\left(w_{-(S \backslash i) \backslash P}^{r}(P, \hat{\pi})\right)_{\hat{\pi} \in \Pi((N \backslash S) \cup i)}\right)$ and the previous equation becomes

$$
w_{-i}^{r}(S \backslash i, \pi)=\sum_{Q \subseteq S \backslash i} \sum_{P \subseteq Q}(-1)^{q-p}\left(w_{-S \backslash P}^{r}\right)(P, \pi) \stackrel{\text { A.1 }}{=} \sum_{Q \subseteq S \backslash i} \eta_{Q, \pi}^{r}{ }_{-S \backslash Q}\left(w_{-S \backslash Q}^{r}\right) \quad \text { Q.E.D. }
$$

\section{Appendix B. Proof of Proposition 3}

By Lemma 10, (ii) implies (i). Now, suppose $w$ and $\tilde{w}$ are such that (i) holds true. We establish (ii) by induction on $|S|$. Induction basis: Let $|S|=1$. Then, $w(i, \pi)-w_{-i}^{r}(\emptyset, \pi) \stackrel{\text { A.2. }}{=}$ $\eta_{i, \pi}^{r}(w) \stackrel{(\mathrm{i})}{=} \eta_{i, \pi}^{r}(\tilde{w}) \stackrel{[\mathrm{A.2}}{=} \tilde{w}(i, \pi)-\tilde{w}_{-i}^{r}(\emptyset, \pi)$. Induction hypothesis $(I H):$ (i) $\Rightarrow($ ii) if $|S| \leq \ell$. Induction step: Let $|S|=\ell+1$. We get

$$
\begin{aligned}
& 0 \stackrel{(\mathrm{i})}{=} w(S, \pi)-w_{-i}^{r}(S \backslash i, \pi)-\left(\tilde{w}(S, \pi)-\tilde{w}_{-i}^{r}(S \backslash i, \pi)\right) \\
& \stackrel{\stackrel{\text { A..2 }}{=}}{\sum_{T \subseteq S: T \ni i}} \eta_{T, \pi}^{r}\left(w_{-S \backslash T}^{r}\right)-\sum_{T \subseteq S: T \ni i} \eta_{T, \pi}^{r}\left(\tilde{w}_{-S \backslash T}^{r}\right) \\
& \stackrel{I H}{=} \eta_{S, \pi}^{r}(w)-\eta_{S, \pi}^{r}(\tilde{w})
\end{aligned}
$$

which concludes the proof.

Q.E.D. 


\section{Appendix C. Proof of Theorem 4}

Proposition 3 and (5) guarantee that $\mathrm{Sh}^{r}$ satisfies $\mathbf{M}^{r}$. Since $r$ and Sh are anonymous, $\mathrm{Sh}^{r}$ satisfies A. By (4) and Efficiency of Sh, $\mathrm{Sh}^{r}$ satisfies $\mathbf{E}$.

Now let $\varphi$ satisfy $\mathbf{A}, \mathbf{E}$, and $\mathbf{M}^{r}$. We need to show that $\varphi=\operatorname{Sh}^{r}$. By Proposition 3 , a modification of dividends $\eta_{T, \pi}^{r}\left(w_{-S \backslash T}^{r}\right), T \subseteq N \backslash i$, does not alter the payoff of player $i$ according to $\varphi$. In the following, we will make such modifications. The proof proceeds by induction on $\# w$, being the maximal number of players being symmetric to each other in $w$,

$$
\# w \equiv \max _{S \subseteq N: \text { all players in } S \text { are symmetric } w}|S|,
$$

where $j$ and $j^{\prime}$ are symmetric in $w$ if $w(S, \pi)=w(\sigma(S, \pi))$ for any transposition $\sigma$ that swaps $j$ and $j^{\prime}$.

Induction basis: For $\# w=n, \varphi=\mathrm{Sh}^{r}$ follows from $\mathbf{A}$ and $\mathbf{E}, \varphi_{i}(w)=w(N, \emptyset) / n=$ $\operatorname{Sh}_{i}^{r}(w)$ for all $i \in N$. Induction hypothesis: For all $w$ such that $\# w>\ell$, we have $\varphi=\operatorname{Sh}^{r}$.

Induction step: Let $w$ be such that $\# w=\ell$ and let $S^{*}$ denote a set of $\ell$ symmetric players, $\left|S^{*}\right|=\ell$. Since $\ell<n$, there is some $\hat{\imath} \notin S^{*}$. We now manipulate $w$ in a way to make the players in $S^{*}$ symmetric to $\hat{\imath}$ without changing $\hat{\imath}$ 's marginal contributions, i.e., by Proposition 3 , without changing $\eta_{T, \pi}^{r}\left(w_{-S \backslash T}^{r}\right)$ for $T \ni \hat{\imath}$. To this end, we define $\tilde{w}$ via $r$-dividends.

Pick some $\hat{\jmath} \in S^{*}$, and define for all $(S, \pi) \in \mathcal{E}(N)$ such that $S \ni \hat{\jmath}$, and $T \subseteq S$ set $\tilde{\eta}_{T, \pi}^{r} \equiv \eta_{\sigma T, \sigma \pi}^{r}\left(w_{-\sigma S \backslash \sigma T}^{r}\right)$, where $\sigma$ is the transposition that swaps $\hat{\imath}$ and $\hat{\jmath}$, and keeps the other players fixed; and define $\tilde{\eta}_{T, \pi}^{r} \equiv \eta_{T, \pi}^{r}\left(w_{-S \backslash T}^{r}\right)$ for the remaining dividends. Note that $\tilde{\eta}_{T, \pi}^{r} \equiv \eta_{T, \pi}^{r}\left(w_{-S \backslash T}^{r}\right)$ whenever $T \ni \hat{\imath}$. We construct $\tilde{w}$ by

$$
\tilde{w}(S, \pi) \equiv \sum_{T \subseteq S} \tilde{\eta}_{T, \pi}^{r}
$$

It further is clear that the players $\hat{\jmath}$ and $\hat{\imath}$ are symmetric in $\tilde{w}$. Now, we show that the marginal contributions of $\hat{\imath}$ are the same in $\tilde{w}$ as in $w$, because we do not know whether $\eta_{T, \pi}^{r}\left(w_{-S \backslash T}^{r}\right)=\eta_{T, \pi}^{r}\left(\tilde{w}_{-S \backslash T}^{r}\right)$ for $T \ni \hat{\imath}$. If $\hat{\jmath} \notin S$, then $\tilde{w}(S \backslash \hat{\imath}, \hat{\pi})=w(S \backslash \hat{\imath}, \hat{\pi})$ for all $\hat{\pi} \in \Pi((N \backslash S) \cup \hat{\imath})$ so that $\tilde{w}_{-\hat{\imath}}^{r}(S \backslash \hat{\imath}, \pi)=w_{-\hat{\imath}}^{r}(S \backslash \hat{\imath}, \pi)$. Moreover, $\tilde{w}(S, \pi)=\sum_{T \subseteq S} \tilde{\eta}_{T, \pi}^{r}=$ $\sum_{T \subseteq S} \eta_{T, \pi}^{r}\left(w_{-S \backslash T}^{r}\right)=w(S, \pi)$. If $\hat{\imath}, \hat{\jmath} \in S$, we have

$$
\tilde{w}(S, \pi)-\tilde{w}_{-\hat{\imath}}^{r}(S \backslash \hat{\imath}, \pi)=\tilde{w}(S, \pi)-\tilde{w}_{-\hat{\jmath}}^{r}(S \backslash \hat{\jmath}, \pi)
$$

because $\hat{\imath}$ and $\hat{\jmath}$ are symmetric in $\tilde{w}$. Since $\tilde{w}(S \backslash \hat{\jmath}, \hat{\pi})=w(S \backslash \hat{\jmath}, \hat{\pi})$ for all $\hat{\pi} \in \Pi((N \backslash S) \cup \hat{\jmath})$, 
we have $\tilde{w}_{-\hat{\jmath}}^{r}(S \backslash \hat{\jmath}, \pi)=w_{-\hat{\jmath}}^{r}(S \backslash \hat{\jmath}, \pi)$ and we obtain

$$
\begin{aligned}
\tilde{w}(S, \pi)-\tilde{w}_{-\hat{\imath}}^{r}(S \backslash \hat{\imath}, \pi) & =\tilde{w}(S, \pi)-w_{-\hat{\jmath}}^{r}(S \backslash \hat{\jmath}, \pi) \\
& \stackrel{\text { A.2 }}{=} \tilde{w}(S, \pi)-w(S, \pi)+\sum_{T \subseteq S: T \ni \hat{\jmath}} \eta_{T, \pi}^{r}\left(w_{-S \backslash T}^{r}\right) \\
& \stackrel{\text { C. } 1]}{=} \sum_{T \subseteq S} \tilde{\eta}_{T, \pi}^{r}-w(S, \pi)+\sum_{T \subseteq S: T \ni \hat{\jmath}} \eta_{T, \pi}^{r}\left(w_{-S \backslash T}^{r}\right) \\
& \stackrel{3}{=} \sum_{T \subseteq S} \tilde{\eta}_{T, \pi}^{r}-\sum_{T \subseteq S} \eta_{T, \pi}^{r}\left(w_{-S \backslash T}^{r}\right)+\sum_{T \subseteq S: T \ni \hat{\jmath}} \eta_{T, \pi}^{r}\left(w_{-S \backslash T}^{r}\right) .
\end{aligned}
$$

Now $\sum_{T \subseteq S} \tilde{\eta}_{T, \pi}^{r}-\sum_{T \subseteq S} \eta_{T, \pi}^{r}\left(w_{-S \backslash T}^{r}\right)=\sum_{T \subseteq S: T \ni \hat{\jmath}} \tilde{\eta}_{T, \pi}^{r}-\sum_{T \subseteq S: T \ni \hat{\jmath}} \eta_{T, \pi}^{r}\left(w_{-S \backslash T}^{r}\right)$ because $\tilde{\eta}_{T, \pi}^{r}=$ $\eta_{T, \pi}^{r}\left(w_{-S \backslash T}^{r}\right)$ if $\hat{\jmath} \notin T$, and we get

$$
\tilde{w}(S, \pi)-\tilde{w}_{-\hat{\imath}}^{r}(S \backslash \hat{\imath}, \pi)=\sum_{T \subseteq S: T \ni \hat{\jmath}} \tilde{\eta}_{T, \pi}^{r}=\sum_{T \subseteq S: T \ni \hat{\jmath}} \eta_{\sigma T, \sigma \pi}^{r}\left(w_{-\sigma S \backslash \sigma T}^{r}\right) .
$$

Note that $\hat{\imath}, \hat{\jmath} \in S$ means $\sigma \pi=\pi$, and $\sum_{T \subseteq S: T \ni \hat{\jmath}} \eta_{\sigma T, \sigma \pi}^{r}\left(w_{-\sigma S \backslash \sigma T}^{r}\right)$ becomes $\sum_{T \subseteq S: T \ni \hat{\imath}} \eta_{T, \pi}^{r}\left(w_{-S \backslash T}^{r}\right)$. Hence

$$
\tilde{w}(S, \pi)-\tilde{w}_{-\hat{\imath}}^{r}(S \backslash \hat{\imath}, \pi)=\sum_{T \subseteq S: T \ni \hat{\imath}} \eta_{T, \pi}^{r}\left(w_{-S \backslash T}^{r}\right) \stackrel{[3]}{=} w(S, \pi)-w_{-\hat{\imath}}^{r}(S \backslash \hat{\imath}, \pi) .
$$

We can successively apply the above procedure for all $j \in S$, arriving at $\tilde{w}$ in which all players $S^{*} \cup \hat{\imath}$ are symmetric and for which $\tilde{w}(S, \pi)-\tilde{w}_{-\hat{\imath}}^{r}(S \backslash \hat{\imath}, \pi)=w(S, \pi)-w_{-\hat{\imath}}^{r}(S \backslash \hat{\imath}, \pi)$. By $\mathbf{M}^{r}, \varphi_{\hat{\imath}}(\tilde{w})=\varphi_{\hat{\imath}}(w)$. The argument can be repeated for all $i \in N \backslash S^{*}$ so that $\varphi_{i}(w)=\operatorname{Sh}_{i}^{r}(w)$ for all $i \in N \backslash S^{*}$. This also determines the payoff accruing to these players, $\sum_{i \in N \backslash S^{*}} \varphi_{i}(w)=\sum_{i \in N \backslash S^{*}} \operatorname{Sh}_{i}^{r}(w)$ and, using $\mathbf{E}$, it determines the payoffs left for the symmetric players in $S^{*}, \sum_{j \in S^{*}} \varphi_{j}(w)=w(N, \emptyset)-\sum_{i \in N \backslash S^{*}} \varphi_{i}(w)$. Using A, we finally determine their payoffs as $\varphi_{j}(w)=\frac{1}{\# w} \sum_{j \in S^{*}} \varphi_{j}(w)=\frac{1}{\# w} \sum_{j \in S^{*}} \operatorname{Sh}_{j}^{r}(w)=\operatorname{Sh}_{j}^{r}(w)$ for $j \in S^{*}$. Hence, $\varphi=\mathrm{Sh}^{r}$.

Q.E.D.

\section{Appendix D. Proof of Corollary 5}

Clearly, the $r^{\star}$ is linear and anonymous. Because of linearity, it suffices to show path independence for Dirac games, which are defined by

$$
\delta_{T, \tau}(S, \pi)= \begin{cases}1, & \text { if }(S, \pi)=(T, \tau), \\ 0, & \text { else. }\end{cases}
$$

For all $N \in \mathcal{N}, i, j \in N, i \neq j$, and $(T, \tau) \in \mathcal{E}(N), T \neq \emptyset$, we have

$$
\left(\delta_{T, \tau}\right)_{-i}^{\star} \stackrel{(? ? ?)}{=} \begin{cases}\frac{1}{n-t} \delta_{T, \tau_{-i}}, & \text { if } i \in N \backslash T, \tau(i)=\{i\}, \\ \frac{|\tau(i)|}{n-t} \delta_{T, \tau_{-i}} & i \in N \backslash T, \tau(i) \neq\{i\}, \\ \mathbf{0}^{N \backslash i}, & i \in T .\end{cases}
$$


where $\mathbf{0}^{N \backslash i} \in \mathbb{V}(N \backslash i)$ denotes the null game. If $i \in T$ or $j \in T$, we have $\left(\left(\delta_{T, \tau}\right)_{-i}^{\star}\right)_{-j}^{\star} \stackrel{\text { D.1 }}{=}$ $\mathbf{0}^{N \backslash\{i, j\}}$. For $i, j \in N \backslash T$, we get

$$
\left(\left(\delta_{T, \tau}\right)_{-i}^{\star}\right)_{-j}^{\star} \stackrel{\text { D.1 }}{=} \begin{cases}\frac{1}{(n-t)(n-1-t)} \delta_{T, \tau \backslash\{i, j\}}, & \tau(i)=\tau(j)=\{i, j\}, \\ \frac{|\tau(i)|(|\tau(i)|-1)}{(n-t)(n-1-t)} \delta_{T, \tau_{-\{i, j\}},}, & \tau(i)=\tau(j) \neq\{i, j\}, \\ \frac{1}{(n-t)(n-1-t)} \delta_{T, \tau_{-\{i, j\}},}, & \tau(i) \neq \tau(j), \tau(i)=\{i\}, \tau(j)=\{j\}, \\ \frac{|\tau(i)|}{(n-t)(n-1-t)} \delta_{T, \tau_{-\{i, j\}},}, & \tau(i) \neq \tau(j), \tau(i)=\{i\}, \tau(j) \neq\{j\}, \\ \frac{|\tau(i)||\tau(j)|}{(n-t)(n-1-t)} \delta_{T, \tau_{-\{i, j\}},}, & \tau(i) \neq \tau(j), \tau(i) \neq\{i\}, \tau(j)=\{j\}, \\ \delta_{T, \tau_{-\{i, j\}},}, & \tau(i) \neq \tau(j), \tau(i) \neq\{i\}, \tau(j) \neq\{j\} .\end{cases}
$$

Hence, the order of removing $i$ and $j$ from the game does not matter.

Q.E.D.

\section{Appendix E. Proof of Lemma 7}

Note that for given $(T, \tau)$ and $S \supseteq T$, there is only a single partition $\pi=\tau_{-S \backslash T}$ such that $(T, \tau) \leq(S, \pi)$. Alonso Meijide et al. (2019, Proposition 4.1) use this and show that the Möbius inverse for $\leq$ is calculated in a similar way as dividends for TU games,

$$
m_{w}^{\leq}(T, \tau)=\sum_{\left(S^{\prime}, \pi^{\prime}\right) \leq(T, \tau)}(-1)^{t-s^{\prime}} w\left(S^{\prime}, \pi^{\prime}\right) .
$$

Insertion into the definition of $w_{-i}^{\leq}(S \backslash i, \pi),(13)$, gives

$$
\begin{aligned}
w_{-i}^{\leq}(S \backslash i, \pi) & =\sum_{(T, \tau):(T, \tau) \leq(S, \pi), T \subseteq S \backslash i} \sum_{\left(S^{\prime}, \pi^{\prime}\right) \leq(T, \tau)}(-1)^{t-s^{\prime}} w\left(S^{\prime}, \pi^{\prime}\right) \\
& =\sum_{\left(S^{\prime}, \pi^{\prime}\right) \leq(S, \pi)} \sum_{T: S^{\prime} \subseteq T \subseteq S \backslash i} \sum_{\tau \in \Pi(N \backslash T): \pi_{-T \backslash S^{\prime}}^{\prime}=\tau, \tau-S \backslash T}=\pi \\
& =\sum_{\left(S^{\prime}, \pi^{\prime}\right) \leq(S, \pi)} \sum_{T: S^{\prime} \subseteq T \subseteq S \backslash i}(-1)^{t-s^{\prime}} w\left(S^{\prime}, \pi^{\prime}\right)
\end{aligned}
$$

The last term vanishes unless $S^{\prime}=S \backslash i$, because

$$
\sum_{T: S^{\prime} \subseteq T \subseteq S \backslash i}(-1)^{t-s^{\prime}}=\sum_{t=s^{\prime}}^{s-1}(-1)^{t-s^{\prime}}\left(\begin{array}{c}
s-1-s^{\prime} \\
t-s^{\prime}
\end{array}\right)=\left\{\begin{array}{cc}
0 & s \neq s^{\prime}-1 \\
1 & s=s^{\prime}-1
\end{array}\right.
$$

so that

$$
w_{-i}^{\leq}(S \backslash i, \pi)=\sum_{\left(S \backslash i, \pi^{\prime}\right) \leq(S, \pi)} w\left(S \backslash i, \pi^{\prime}\right)=\sum_{\pi^{\prime} \in \Pi((N \backslash S) \cup i): \pi_{-i}^{\prime}=\pi} w\left(S \backslash i, \pi^{\prime}\right) .
$$




\section{Appendix F. Proof of Theorem 8}

First we establish that $\mathcal{U}=\left\{u_{T, \tau}^{\leq}\right\}_{(T, \tau) \in \mathcal{E}(N)}$ is a basis of $\mathbb{W}(N)$ with coefficients $\left\{m_{w}^{\leq}(T, \tau)\right\}_{(T, \tau) \in \mathcal{E}(N)}$. For all $(S, \pi) \in \mathcal{E}(N)$, we get

$$
\begin{aligned}
& \sum_{(T, \tau) \in \mathcal{E}(N)} m_{w}^{\leq}(T, \tau) u_{T, \tau}(S, \pi) \stackrel{10 p}{=} m_{w}^{\leq}(S, \pi)+\sum_{(T, \tau) \nsupseteq(S, \pi)} m_{w}^{\leq}(T, \tau) \\
& \stackrel{122}{=} w(S, \pi)-\sum_{\left(S^{\prime}, \pi^{\prime}\right) \ngtr(S, \pi)} m_{w}^{\leq}\left(S^{\prime}, \pi^{\prime}\right)+\sum_{(T, \tau) \ngtr(S, \pi)} m_{w}^{\leq}(T, \tau) \\
& =w(S, \pi) \text {. }
\end{aligned}
$$

Thus, $\mathcal{U}$ spans $\mathbb{W}(N)$, and since $\operatorname{dim} \mathcal{E}(N)=|\mathcal{E}(N)|=|\mathcal{U}|, \mathcal{U}$ is a basis of $\mathcal{E}(N)$. Consequently, the coefficients $m_{w}^{\leq}(T, \tau)$ are the unique coefficients such that $w=\sum_{(T, \tau) \in \mathcal{E}(N)} m_{w}^{\leq}(T, \tau) u_{\bar{T}, \tau}^{\leq}$. Hence,

$$
\operatorname{Sh}_{i}^{\leq}\left(u_{T, \tau}^{\leq}\right)=\sum_{(T, \tau) \in \mathcal{E}(N): T \ni i} m_{u_{T, \tau}^{\leq}}^{\leq}(T, \tau)=\frac{1}{t},
$$

and consequently

$$
\sum_{i \in N} \operatorname{Sh}_{i}^{\leq}(w)=\sum_{i \in N} \sum_{(T, \tau) \in \mathcal{E}(N): T \ni i} m_{w}^{\leq}(T, \tau) \frac{1}{t}=\sum_{(T, \tau) \in \mathcal{E}} m_{w}^{\leq}(T, \tau) \stackrel{\sqrt[12]{=}}{=} w(N, \emptyset),
$$

i.e., $\mathrm{Sh}^{\leq}$satisfies E. Moreover, it is clear from the definition that $\mathrm{Sh}^{\leq}$satisfies A. Now let $\left(^{*}\right) w(S, \pi)-\tilde{w}(S, \pi)=w_{-i}^{\leq}(S \backslash i, \pi)-\tilde{w}_{-i}^{\leq}(S \backslash i, \pi)$ for all $S \ni i$ and $\pi \in \Pi(N \backslash S)$. Then,

$$
\operatorname{Sh}_{i}^{\leq}(w)-\operatorname{Sh}_{i}^{\leq}(\tilde{w})=\sum_{(T, \tau) \in \mathcal{E}(N): T \ni i} \frac{1}{t}\left(m_{w}^{\leq}(T, \tau)-m_{\tilde{\tilde{w}}}^{\leq}(T, \tau)\right)
$$

and since $T \ni i$ implies

$$
\begin{aligned}
m_{w}^{\leq}(T, \tau)-m_{\tilde{\tilde{w}}}(T, \tau) & \stackrel{(12)}{=} w(T, \tau)-\sum_{(S, \pi) \ddagger(T, \tau)} m_{w}^{\leq}(S, \pi)-\left(\tilde{w}(T, \tau)-\sum_{(S, \pi) \ddagger(T, \tau)} m_{\tilde{\tilde{w}}}^{\leq}(S, \pi)\right) \\
& \stackrel{(13)}{=} w(S, \pi)-w_{-i}^{\leq}(S \backslash i, \pi)-\left(\tilde{w}(S, \pi)-\tilde{w}_{-i}^{\leq}(S \backslash i, \pi)\right) \\
& \stackrel{(*)}{=} 0,
\end{aligned}
$$

we get $\operatorname{Sh}_{i}^{\leq}(w)=\operatorname{Sh}_{i}^{\leq}(\tilde{w})$. With Lemma 7, we find that $\mathrm{Sh}^{\leq}$satisfies $\mathbf{M} \leq$. It remains to show that 13 defines a path independent restriction. It suffices to show this for $u_{T, \tau}^{\leq}$. We have

$$
\left(u_{\bar{T}, \tau}^{\leq}\right)_{-i}^{\leq} \stackrel{13}{=} \begin{cases}u_{T, \tau_{-i}}^{\leq}, & \text {if } i \in N \backslash T, \tau(i)=\{i\} \\ \mathbf{0}, & i \in T .\end{cases}
$$

Thus, if $i \in T$ or $j \in T$, then $\left(\left(u_{T, \tau}^{\leq}\right)_{-i}^{\leq}\right)_{-j}^{\leq}=\mathbf{0}=\left(\left(u_{\bar{T}, \tau}^{\leq}\right)_{-j}^{\leq}\right)_{-i}^{\leq}$. If $\{i, j\} \in N \backslash T$, then $\left(\left(u_{\bar{T}, \tau}^{\leq}\right)_{-i}^{\leq}\right)_{-j}^{\leq}=u_{\bar{T}, \tau_{-i j}}^{\leq}=\left(\left(u_{\bar{T}, \tau}^{\leq}\right)_{-j}^{\leq}\right)_{-i}^{\leq}$. 


\section{References}

Albizuri, M. J., Arin, J., Rubio, J., 2005. An axiom system for a value for games in partition function form. International Game Theory Review 7, 63-73.

Alonso Meijide, J. M., Alvarez-Mozos, M., Gloria Fiestras-Janeiro, M., Jimenez-Losada, A., 01 2019. A new order on embedded coalitions: Properties and applications. SSRN Electronic Journal.

Bolger, E. M., 1989. A set of axioms for a value for partition function games. International Journal of Game Theory 18, 37-44.

van den Brink, R., 2007. Null or nullifying players: The difference between the Shapley value and equal division solutions. Journal of Economic Theory 136, 767-775.

de Clippel, G., Serrano, R., 2008. Marginal contributions and externalities in the value. Econometrica 76 (6), 1413-1436.

Dutta, B., Ehlers, L., Kar, A., 2010. Externalities, potential, value and consistency. Journal of Economic Theory 145 (6), 2380-2411.

Harsanyi, J. C., 1959. A bargaining model for cooperative $n$-person games. In: Tucker, A. W., Luce, R. D. (Eds.), Contributions to the Theory of Games IV. Vol. 2. Princeton University Press, Princeton NJ, pp. 325-355.

Hu, C.-C., Yang, Y.-Y., 2010. An axiomatic characterization of a value for games in partition function form. SERIEs 1 (4), 475-487.

Lucas, W. F., Thrall, R. M., 1963. n-person games in partition function form. Naval Research Logistics Quarterly X, 281-298.

Macho-Stadler, I., Pérez-Castrillo, D., Wettstein, D., 2007. Sharing the surplus: An extension of the Shapley value for environments with externalities. Journal of Economic Theory 135 (1), 339-356.

McQuillin, B., 2009. The extended and generalized Shapley value: Simultaneous consideration of coalitional externalities and coalitional structure. Journal of Economic Theory 144, 696-721.

Milnor, J., 1952. Reasonable outcomes for $n$-person games. Tech. Rep. RM-916, RAND Corporation.

Moulin, H., 1988. Axioms of cooperative decision making. Cambridge Universitiy Press, New York.

Pham Do, K. H., Norde, H., 2007. The Shapley value for partition function form games. International Game Theory Review 9, 353-360.

Pintér, M., 2015. Young's axiomatization of the Shapley value: a new proof. Annals of Operations Research 235 (1), 665-673.

Shapley, L. S., 1953. A value for $n$-person games. In: Kuhn, H., Tucker, A. (Eds.), Contributions to the Theory of Games. Vol. II. Princeton University Press, Princeton, pp. 307-317.

Skibski, O., Michalak, T. P., Woolbridge, M., 2018. The stochastic Shapley value for coalitional games with externalities. Games and Economic Bahavior 108, 65-80.

Young, H. P., 1985. Monotonic solutions of cooperative games. International Journal of Game Theory 14, $65-72$. 


\section{Recent ESMT Working Papers}

ESMT No.

Consumer choice under limited attention when alternatives have different

$16-04(R 3)$ information costs

Frank Huettner, ESMT Berlin

Tamer Boyacı, ESMT Berlin

Yalçın Akçay, Melbourne Business School

Opaque queues: Service systems with rationally inattentive customers

Caner Canyakmaz, ESMT Berlin

Tamer Boyaci, ESMT Berlin

The Coleman-Shapley-index: Being decisive within the coalition of the interested

André Casajus, HHL Leipzig Graduate School of Management

Frank Huettner, ESMT Berlin

Reverse privatization as a reaction to the competitive environment: Evidence from solid waste collection in Germany

Juri Demuth, E.CA Economics

Hans W. Friederiszick, ESMT Berlin and E.CA Economics

Steffen Reinhold, E.CA Economics

Knowing me, knowing you: Inventor mobility and the formation of technologyoriented alliances

Stefan Wagner, ESMT Berlin

Martin C. Goossen, Tilburg University 